\title{
State-Space Modeling and Active Vibration Control of Smart Flexible Cantilever Beam with the Use of Finite Element Method
}

\author{
Kerim Gokhan Aktas \\ Department of Mechanical Engineering \\ Karabuk University \\ Karabuk, Turkey \\ kerimgokhanaktas@karabuk.edu.tr
}

\author{
Ismail Esen \\ Department of Mechanical Engineering \\ Karabuk University \\ Karabuk, Turkey \\ iesen@karabuk.edu.tr
}

\begin{abstract}
The aim of this study is to design a Linear Quadratic Regulator (LQR) controller for the active vibration control of a smart flexible cantilever beam. The mathematical model of the smart beam was created on the basis of the Euler-Bernoulli beam theory and the piezoelectric theory. State-space and finite element models used in the LQR controller design were developed. In the finite element model of the smart beam containing piezoelectric sensors and actuators, the beam was divided into ten finite elements. Each element had two nodes and two degrees of freedom were defined for each node, transverse displacement, and rotation. Two Piezoelectric ceramic lead Zirconate Titanate (PZT) patches were affixed to the upper and lower surfaces of the beam element as pairs of sensors and actuators. The location of the piezoelectric sensor and actuator pair changed and they were consecutively placed on the fixed part, the middle part, and the free end of the beam. In each case, the design of the LQR controller was made considering the first three dominant vibratory modes of the beam. The effect of the position of the sensor-actuator pair on the beam on the vibration damping capability of the controller was investigated. The best damping performance was found when the sensor-actuator pair was placed at the fixed end.
\end{abstract}

Keywords-vibration control; piezoelectric; LQR control; finite element analysis; smart beam

\section{INTRODUCTION}

Because of the low resistance of lightweight and flexible structures, vibration problems occur at low frequencies and their control is a major problem. Active vibration control systems are preferred because passive methods are considered inadequate in dealing with this problem. One of the most effective solutions of the vibration problem in light and flexible structures is to make them smart using smart materials [1]. Piezoelectric materials come to the forefront in smart materials due to advantages such as high sensitivity in sensing and drive, being applicable in a wide frequency range and being supplied in different shapes and sizes. Piezoelectric materials have been widely used recently in various areas especially in aviation, flexible robot arms, energy harvesting, and noise and shape control due to their ability to convert mechanical into electrical and electrical into mechanical energy [2-5]. Flexible beam elements are distributed parameter systems with infinite Degrees of Freedom (DoF). The active vibration control generally requires a low-order mathematical model of the system containing a limited number of DoF [6]. In particular, care must be taken so that the control of low-frequency modes does not affect the stabilization of high-grade modes, since sensors and actuators have limited bandwidth [7, 8]. Many techniques have been used to solve this problem, but the most effective is the use of collocated sensors and actuators that work in harmony with each other [9]. Vibration can be controlled by different control techniques. The most used control techniques are classical control algorithms such as Positive Position Feedback (PPF), velocity feedback and PID control [10-14]. It is very important to specify the sensor and actuator position optimally to keep the control capability at maximum. Otherwise, control spillover and observation spillover may occur, and this will affect the efficiency of the control system [6].

Authors in [15] proposed a controller combining PPF and PD control methods to suppress the first three vibration modes of the cantilever plate. Authors in [16] used two types of actuators (an inertial and a distributed strain actuator) and two types of controllers (PVF and $\mathrm{H} \infty$ control) to suppress the vibration of a fully clamped beam, and found that the best result was obtained with the $\mathrm{H} \infty$ controller and a distributed strain actuator. Authors in [17] designed a LQR controller based on the independent mode space control techniques. LQR control is one of the methods frequently used in active vibration control [18]. In LQR control, the performance index is minimized with optimum weight matrices. The LQR control provides the optimal response between the response speed and energy required to suppress vibration. [Q] and [R] are the most important parameters in keeping the permissible actuator voltage within certain limits while keeping the control performance at maximum level. Therefore, the important thing in LQR control is to determine the optimum state weighting matrix [Q] and the input weighting matrix [R]. Significant research has been done on the determination of weight matrices [19-21]. 
In this study, the method of constructing a comprehensive mathematical model including piezoelectric mass and stiffness sensor-actuator and controlling the first three dominant vibration modes of a cantilever beam using the LQR controller are presented. The collocated piezoelectric sensor and actuator pair were placed on various finite elements. This allowed us to examine the effect of the sensor-actuator pair position on the performance of the controller. Since piezoelectric actuators have a certain operating voltage range, it is important to keep the actuator voltage within the allowable limits for a realistic control performance. For this reason, unlike most studies in the literature, the necessary actuator voltage was determined while ensuring optimum controller performance. As a result of the simulation study, system response and required actuator voltage were determined.

\section{SYSTEM MODELING}

In this section, the mathematical model of a smart cantilever beam with a piezoelectric collocated sensor and actuator pair is presented. The mathematical model of the smart beam was created on the basis of the Euler-Bernoulli beam theory. As shown in Figures 1 and 2, the beam is divided into 10 finite elements, each element with two nodes and two DoF were defined for each node: transverse displacement and rotation $\left(v_{1}, \theta_{1}, v_{2}, \theta_{2}\right)$. The physical and mechanical properties of the beam and PZT patches are given in Table I.

TABLE I. MECHANICAL AND PHYSICAL PROPERTIES

\begin{tabular}{|l|c|c|}
\hline \multicolumn{1}{|c|}{ Parameters } & Beam & PZT Patches \\
\hline Length & $L_{b}=0.25 \mathrm{~m}$ & $l p=0.025 \mathrm{~m}$ \\
\hline Width & $w=0.03 \mathrm{~m}$ & $w=0.03 \mathrm{~m}$ \\
\hline Thickness & $h_{b}=1.0 \mathrm{~mm}$ & $h_{a}=\mathrm{h}_{\mathrm{s}}=0.55 \mathrm{~mm}$ \\
\hline Density & $\rho_{b}=8030 \mathrm{~kg} / \mathrm{m}^{3}$ & $\rho_{p}=7700 \mathrm{~kg} / \mathrm{m}^{3}$ \\
\hline Young's modulus & $\mathrm{E}_{\mathrm{b}}=193 \mathrm{GPa}$ & $E_{p}=68 \mathrm{GPa}$ \\
\hline PZT strain constant & & $d_{31}=125 \times 10^{-12} \mathrm{~m} / \mathrm{V}$ \\
\hline
\end{tabular}

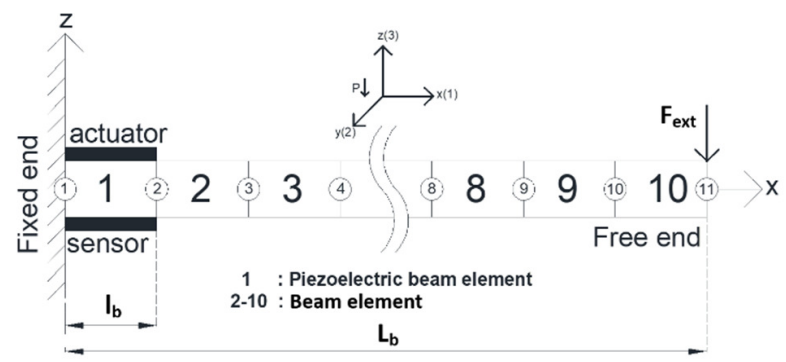

Fig. 1. Finite element discretization of the smart cantilever beam.

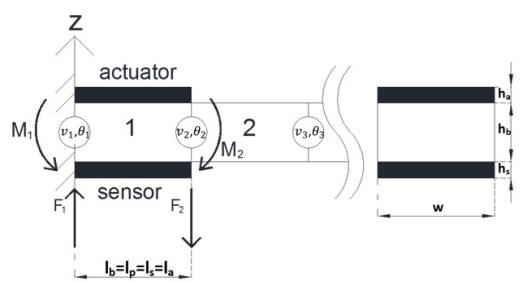

Fig. 2. Beam element and piezoelectric beam element.

As shown in Figure 2, the section of the beam without the piezoelectric patch is expressed as a beam element, and the section with the piezoelectric patch is expressed as a piezoelectric beam element. In Figures $1-2, F_{\text {ext }}, L_{b}, l_{b}, l_{p}\left(l_{s}, l_{a}\right)$, and $P$ represent the external disruptive force applying at the free end of the beam, the length of the entire beam, the length of the beam element, the length of the piezoelectric sensor and of the actuator patch, and the polarization direction.

\section{A. Modeling of the Beam Element}

In the modeling, beam and piezoelectric patches were considered as an Euler-Bernoulli beam. The governing equations of motion of the smart beam for forced motion can be written as a fourth order partial differential equation [22] :

$$
\rho A \frac{\partial^{2} v(x, t)}{\partial t^{2}}+E I \frac{\partial^{4} v(x, t)}{\partial x^{4}}=F_{\text {ext }}
$$

where $v$ is the displacement of the beam, $\rho$ is the mass density, $A$ is the cross-section area, $E$ is the Young's Modulus, $I$ is the moment of inertia, and $F_{\text {ext }}$ is the external force. In order to obtain the shape functions, the beam displacement function is taken as a cubic displacement function as in (2):

$$
v(x, t)=a_{1} x^{3}+a_{2} x^{2}+a_{3} x+a_{4}
$$

where $a_{i}$ indicates the total degree of freedom, including displacement and rotation in each element $\left(v_{1}, \theta_{1}, v_{2}, \theta_{2}\right)$. Constants $a_{1}, a_{2}, a_{3}$ and $a_{4}$ are obtained by using cantilever beam boundary conditions.

Substituting the constants $\left(a_{1}, a_{2}, a_{3}, a_{4}\right)$ in (2), the displacement function can be obtained as:

$$
v(x, t)=\left[\begin{array}{llll}
N_{1}(x) & \ldots & \ldots & N_{4}(x)
\end{array}\right]\left[\begin{array}{l}
v_{1} \\
\theta_{1} \\
v_{2} \\
\theta_{2}
\end{array}\right]=[N][p]
$$

where $[N]$ represents the shape function and $[p]$ represents the nodal displacement vector. The strain energy in bending and bending moment can be found as [23] :

$$
U=\int_{0}^{L} \frac{M^{2}}{2 E I} d x, M=E I \frac{\partial^{2} v}{\partial x^{2}}
$$

Using (4), the potential energy is obtained as in (5):

$$
\begin{gathered}
U=\frac{E_{b} I_{b}}{2} \int_{0}^{l_{b}}\left[\frac{\partial^{2} v}{\partial x^{2}}\right]^{2} d x=\frac{E_{b} I_{b}}{2} \int_{0}^{l_{b}}[p]^{T}\left[n_{2}\right]^{T}\left[n_{2}\right][p] d x \\
=\frac{1}{2}[p]^{T}\left[E_{b} I_{b} \int_{0}^{l_{b}}\left[n_{2}\right]^{T}\left[n_{2}\right] d x\right]
\end{gathered}
$$

The kinetic energy can be found as:

$$
\begin{gathered}
T=\frac{\rho_{b} A_{b}}{2} \int_{0}^{l_{b}}\left[\frac{\partial v}{\partial t}\right]^{2} d t=\frac{\rho_{b} A_{b}}{2} \int_{0}^{l_{b}}[\dot{p}]^{T}[n]^{T}[n][\dot{p}] d t \\
=\frac{1}{2}[\dot{p}]^{T}\left[\rho_{b} A_{b} \int_{0}^{l_{b}}[n]^{T}[n] d t\right][\dot{p}]
\end{gathered}
$$

where $n_{2}$ and $n$ are the second spatial derivative and the time derivative of the shape function, respectively. Total kinetic and potential energy can be found as:

$$
\begin{aligned}
U & =\frac{1}{2}[p]^{T}\left[k_{b}\right][p] \\
T & =\frac{1}{2}[\dot{p}]^{T}\left[m_{b}\right][\dot{p}]
\end{aligned}
$$

Using the Lagrange equations, the stiffness and mass matrix of each beam element can be written as in (9) and (10): 


$$
\begin{gathered}
{\left[k_{b}\right]=\frac{E_{b} I_{b}}{l_{b}{ }^{3}}\left[\begin{array}{cccc}
12 & 6 l_{b} & -12 & 6 l_{b} \\
6 l_{b} & 4 l_{b}{ }^{2} & -6 l_{b} & 2 l_{b}{ }^{2} \\
-12 & -6 l_{b} & 12 & -6 l_{b} \\
6 l_{b} & 2 l_{b}{ }^{2} & -6 l_{b} & 4 l_{b}{ }^{2}
\end{array}\right] \quad(9)} \\
{\left[m_{b}\right]=\frac{\rho_{b} A_{b} l_{b}}{420}\left[\begin{array}{cccc}
156 & 22 l_{b} & 54 & -13 l_{b} \\
22 l_{b} & 4 l_{b}{ }^{2} & 13 l_{b} & -3 l_{b}{ }^{2} \\
54 & 13 l_{b} & 156 & -22 l_{b} \\
-13 l_{b} & -3 l_{b}{ }^{2} & -22 l_{b} & 4 l_{b}{ }^{2}
\end{array}\right]}
\end{gathered}
$$

B. Modeling of the Piezoelectric Beam Element and of the Smart Beam Element

Similarly, when the piezoelectric patches are considered as Euler-Bernoulli beam elements, the stiffness and mass matrix of the piezoelectric patches can be obtained as:

$$
\begin{gathered}
{\left[k_{p}\right]=\frac{E_{p} I_{p}}{l_{p}{ }^{3}}\left[\begin{array}{cccc}
12 & 6 l_{p} & -12 & 6 l_{p} \\
6 l_{p} & 4 l_{p}{ }^{2} & -6 l_{p} & 2 l_{p}{ }^{2} \\
-12 & -6 l_{p} & 12 & -6 l_{p} \\
6 l_{p} & 2 l_{p}{ }^{2} & -6 l_{p} & 4 l_{p}{ }^{2}
\end{array}\right] \quad(11)} \\
{\left[m_{p}\right]=\frac{\rho_{p} A_{p} l_{p}}{420}\left[\begin{array}{cccc}
156 & 22 l_{p} & 54 & -13 l_{p} \\
22 l_{p} & 4 l_{p}{ }^{2} & 13 l_{p} & -3 l_{p}{ }^{2} \\
54 & 13 l_{p} & 156 & -22 l_{p} \\
-13 l_{p} & -3 l_{p}{ }^{2} & -22 l_{p} & 4 l_{p}{ }^{2}
\end{array}\right]}
\end{gathered}
$$

As shown in Figure 2, two piezoelectric patches are bonded to the upper and lower surface of the beam element as a sensor and actuator. This state of the beam is called a smart beam element. The stiffness of a smart beam element can be obtained by the equivalent flexural stiffness method [24] .

$$
(E I)_{e q}=E_{b} I_{b}+2 E_{P} I_{P}
$$

With the help of the parallel axis theorem, the moment of inertia of the smart beam element can be obtained as:

$$
I_{P}=\frac{w h_{p}{ }^{3}}{12}+w h_{P} \frac{\left(h_{p}+h_{b}\right)^{2}}{4}
$$

The mass per unit length of the beam element can be found as:

$$
\rho A=w\left(\rho_{b} h_{b}+2 \rho_{p} h_{p}\right)
$$

where $h_{p}$ and $h_{b}$ represent the thickness of the PZT patch and core beam respectively. The elemental stiffness and mass matrix of the smart beam can be written as:

$$
\begin{gathered}
{\left[k_{s}\right]=\frac{(E I)_{e q}}{l_{s}{ }^{3}}\left[\begin{array}{cccc}
12 & 6 l_{s} & -12 & 6 l_{s} \\
6 l_{s} & 4 l_{s}{ }^{2} & -6 l_{s} & 2 l_{s}{ }^{2} \\
-12 & -6 l_{s} & 12 & -6 l_{s} \\
6 l_{s} & 2 l_{s}{ }^{2} & -6 l_{s} & 4 l_{s}{ }^{2}
\end{array}\right]} \\
{\left[m_{s}\right]=\frac{\rho A l_{s}}{420}\left[\begin{array}{cccc}
156 & 22 l_{s} & 54 & -13 l_{s} \\
22 l_{s} & 4 l_{s}{ }^{2} & 13 l_{s} & -3 l_{s}{ }^{2} \\
54 & 13 l_{s} & 156 & -22 l_{s} \\
-13 l_{s} & -3 l_{s}{ }^{2} & -22 l_{s} & 4 l_{s}{ }^{2}
\end{array}\right]}
\end{gathered}
$$

\section{PIEZOELECTRIC CONSTITUTIVE EQUATIONS}

Piezoelectric materials can be used as both sensors and actuators. If used as an actuator, the piezoelectric patch undergoes mechanical strain when voltage is applied as input (inverse piezoelectric effect). If used as a sensor, the patch generates voltage as an output when mechanical stress is applied to it (direct piezoelectric effect) [25]. When a disruptive external force is applied to the beam, the beam is exposed to vibration. In the vibration damping process, mechanical stress occurs in the beam exposed to vibration, and the patch used as a sensor produces a voltage against this stress. The generated voltage is applied to the controller after the signal conditioning process. After the signal is evaluated, it is applied to the actuator as a control signal. The patch used as actuator undergoes a mechanical strain and suppresses vibration by producing an equal amplitude and an opposite response to vibration. The IEEE piezoelectricity standard was taken into account in defining the electromechanical properties of the piezoelectric material and the piezoelectric material is considered to exhibit linear behavior [26]. The electromechanical equations of linear piezoelectric materials can be written as:

$$
\begin{aligned}
& \varepsilon_{x}=S_{11}{ }^{E} \sigma_{x}+d_{31} E_{z} \\
& D_{z}=d_{31} \sigma_{x}+\xi_{33}{ }^{\sigma} E_{z}
\end{aligned}
$$

where $\varepsilon_{x}, S_{11}, \sigma_{x}, d_{31}, E_{z}, D_{z}$ and $\xi_{33}$ represent the strain $(\mathrm{m} / \mathrm{m})$, compliance $\left(\mathrm{m}^{2} / \mathrm{N}\right)$, stress $\left(\mathrm{N} / \mathrm{m}^{2}\right)$, piezoelectric strain constant $(\mathrm{m} / \mathrm{V})$, electric field $(\mathrm{V} / \mathrm{m})$, electric displacement $\left(\mathrm{C} / \mathrm{m}^{2}\right)$, and permittivity $(\mathrm{F} / \mathrm{m})$. Also, the superscripts $E$ and $\sigma$ represent the constant electric field and the constant voltage.

\section{A. Sensor Equations}

Equation (19) expresses the direct piezoelectric effect and is used to determine the total charge obtained from the PZT sensor. Since no extra electric field is given to the sensor, (19) can be written as:

$$
D_{z}=d_{31} \sigma_{x}=d_{31} C_{11} \varepsilon_{x}=e_{31} \varepsilon_{x}
$$

where $e_{31}$ is the piezoelectric stress/charge constant and $C_{11}$ is the inverse of compliance $\left(1 / S_{11}\right)$. The total charge obtained from the sensor layer can be calculated as:

$$
Q(t)=\int_{A} D_{z} d A
$$

The charge can be converted to current as:

$$
i(t)=\frac{d Q(t)}{d t}=\frac{d}{d t} \int_{A} D_{z} d A=\frac{d}{d t} \int_{A} e_{31} \varepsilon_{x} d A
$$

As we know, strain at any point can be found as [23]:

$$
\varepsilon_{x}=-\frac{z}{R}, \frac{1}{R}=\frac{d^{2} v}{d x^{2}}, \quad \varepsilon_{x}=z \frac{d^{2} v}{d x^{2}}
$$

where $z$ is the distance to the neutral axis and $R$ is the radius of curvature. Equation (22) can be written as:

$$
\begin{gathered}
i(t)=\frac{d}{d t} \int_{A} e_{31} z n_{2} p d A=z e_{31} w \int_{0}^{l_{p}} n_{2} \dot{p} d x \\
z=\left(\frac{h_{b}}{2}+h_{a}\right)
\end{gathered}
$$


The current obtained from the sensor is converted into voltage with the signal conditioning device. The voltage value obtained is given to the actuator with a suitable control gain:

$$
V^{s}(t)=H z e_{31} w \int_{0}^{l_{p}} n_{2} \dot{p} d x=S \dot{p}
$$

where $H$ is the signal conditioning device gain. This sensor voltage is given as input to the controller and the controller output is multiplied by the controller gain and is given to the actuator. The actuator input voltage can be found as:

$$
V^{a}(t)=u=K x V^{s}(t)
$$

where $K$ is the control gain.

\section{B. Actuator Equations}

Equation (18) expresses the inverse piezoelectric effect and is used to calculate the total strain. Since no extra stress is given to the actuator, (18) can be written as:

$$
\varepsilon_{x}=d_{31} E_{z}
$$

When an input voltage is applied to the actuator in the polarization direction, the actuator strains in one direction. The stress and electrical field generated in the actuator can be obtained as:

$$
E_{z}=\frac{V^{a}(t)}{h_{a}}, \sigma_{x}=E_{p} \varepsilon_{x}=E_{p} d_{31} \frac{V^{a}(t)}{h_{a}}
$$

where $E_{p}$ and $h_{a}$ are the Young's Modulus and the thickness of the actuator respectively. As a result of the stress, the bending moment occurs in the beam element. The bending moment in a small cross section can be written as:

$$
d M_{a c t}=E_{p} I_{p} \frac{d^{2} v}{d x^{2}}
$$

The sum of the moments of the stresses on the $x$ axis relative to the $y$ axis can be written as:

$$
M_{a c t}=\int \overline{\bar{z}} \sigma_{x} d z, \quad \overline{\bar{z}}=\left(\frac{h_{a}+h_{b}}{2}\right)
$$
as:

By using (29) and (30), the bending moment can be found

$$
M_{a c t}=E_{p} d_{31} \overline{\bar{z}} V^{a}(t)
$$

The control force applied by the actuator to the beam element can be obtained using (27) and (32):

$$
f_{a c t}=E_{p} d_{31} w \bar{z} \int_{l_{p}} n_{1}{ }^{T} d x V^{a}(t)=G V^{a}(t)
$$

where $G$ is a constant vector of size $(4 \times 1)$.

\section{EQUATIONS OF MOTION, STATE SPACE REPRESENTATION, AND MODEL VERIFICATION}

\section{A. Equations of Motion for the Smart Beam}

The dynamic equation of a smart beam can be written as:

$$
[M]\{\ddot{p}\}+[K]\{p\}=F_{\text {ext }}+F_{\text {ctrl }}
$$

Rayleigh proportional damping method is used to include the damping effect in the model. Structural damping matrix can be obtained as [27]:

$$
C=\alpha M+\beta K
$$

where $C$ is the damping matrix, $\alpha$ is the mass proportional damping coefficient, and $\beta$ is the stiffness proportional damping coefficient. In obtaining the damping matrix of the smart beam, $\alpha$ and $\beta$ coefficients were taken as 0.001 and 0.0001 respectively. $M, C$ and $K$ represent the global mass and the damping and stiffness matrices of each finite element. The mass and stiffness matrices of the entire beam are obtained by combining the local mass and stiffness matrices using the finite element technique. The equation of motion of cantilever beam and sensor output equation can be written as:

$$
\begin{gathered}
{[M]\{\ddot{p}\}+[C]\{\dot{p}\}+[K]\{p\}=F_{\text {ext }}+F_{\text {act }}} \\
y(t)=V^{s}(t)=S \dot{p}
\end{gathered}
$$

In order to examine the dominant first three vibration modes of the system, $p=\Phi d$ conversion was made and the transition from generalized coordinates to principal coordinates was made [28]. Here, $\Phi$ denotes the modal matrix. Using the transformation $p=\Phi d$, (36) and (37) can be written as:

$$
\begin{array}{r}
M \Phi \ddot{d}+C \Phi \dot{d}+K \Phi d=F_{\text {ext }}+F_{\text {act }} \\
y(t)=V^{s}(t)=S \Phi \dot{d} \quad(39)
\end{array}
$$

By pre-multiplying (38) with $\Phi^{T}$, generalized mass, stiffness and damping matrices can be obtained as:

$$
\begin{gathered}
\Phi^{T} M \Phi \ddot{d}+\Phi^{T} C \Phi \dot{d}+\Phi^{T} K \Phi d=\Phi^{T} F_{\text {ext }}+\Phi^{T} F_{a c t} \\
\bar{M} \ddot{d}+\bar{C} \dot{d}+\bar{K} d=\bar{F}_{\text {ext }}+\bar{F}_{\text {act }}
\end{gathered}
$$

The $\bar{M}, \bar{C}$ and $\bar{K}$ matrices are generalized mass, damping matrix, and stiffness matrix respectively, each $(3 \times 3)$ in size. The generalized external force vector $\bar{F}_{\text {ext }}$ is obtained as:

$$
\bar{F}_{\text {ext }}=\Phi^{T} F_{\text {ext }}=\Phi^{T} f r(t)
$$

where $r(t)$ refers to the external force input to the smart beam. The generalized control force vector is expressed by:

$$
\bar{F}_{\text {act }}=\Phi^{T} F_{a c t}=\Phi^{T} G V^{a}(t)
$$

\section{B. State Space Repsentation of the Smart Beam}

The state and output equation of a dynamic system can be written as:

$$
\begin{aligned}
& \dot{x}=A x+B u \\
& y=C x+D u
\end{aligned}
$$

where $A$ is the system matrix, $B$ is the input matrix, $C$ is the output matrix, $D$ is the direct transmission matrix. $x, u$ and $y$ represent the state vector, input vector and output vector respectively. $x$ is used as the state variable to obtain the state equations. The generalized coordinates in (44) can be written in terms of the state variable as follows:

$$
[d]=\left[\begin{array}{l}
d_{1} \\
d_{2} \\
d_{3}
\end{array}\right]=\left[\begin{array}{l}
x_{1} \\
x_{2} \\
x_{3}
\end{array}\right],[\dot{d}]=\left[\begin{array}{l}
\dot{d}_{1} \\
\dot{d}_{2} \\
\dot{d}_{3}
\end{array}\right]=\left[\begin{array}{l}
x_{4} \\
x_{5} \\
x_{6}
\end{array}\right],[\ddot{d}]=\left[\begin{array}{l}
\dot{x}_{4} \\
\dot{x}_{5} \\
\dot{x}_{6}
\end{array}\right]
$$

The state space form of the (36) can be written as: 


$$
\bar{M}\left[\begin{array}{l}
\dot{x}_{4} \\
\dot{x}_{5} \\
\dot{x}_{6}
\end{array}\right]+\bar{C}\left[\begin{array}{l}
x_{4} \\
x_{5} \\
x_{6}
\end{array}\right]+\bar{K}\left[\begin{array}{l}
x_{1} \\
x_{2} \\
x_{3}
\end{array}\right]=\bar{F}_{\text {ext }}+\bar{F}_{\text {act }}
$$

The state equation form of (47) can be written as:

$$
\begin{gathered}
{\left[\begin{array}{c}
\dot{x}_{1} \\
\dot{x}_{2} \\
\dot{x}_{3} \\
\dot{x}_{4} \\
\dot{x}_{5} \\
\dot{x}_{6}
\end{array}\right]=\left[\begin{array}{cc}
0 & I \\
-\bar{M}^{-1} \bar{K} & -\bar{M}^{-1} \bar{C}
\end{array}\right]\left[\begin{array}{c}
x_{1} \\
x_{2} \\
x_{3} \\
x_{4} \\
x_{5} \\
x_{6}
\end{array}\right]+\left[\begin{array}{c}
0 \\
\bar{M}^{-1} \Phi^{T} G
\end{array}\right] u(t)} \\
+\left[\begin{array}{c}
0 \\
\bar{M}^{-1} \Phi^{T} f
\end{array}\right] r(t)
\end{gathered}
$$
as:

The sensor voltage can be taken as output and is obtained

$$
\begin{aligned}
& y(t)=C^{T} x(t)+D u(t) \\
& y(t)=\left[\begin{array}{ll}
0 & S \Phi
\end{array}\right]\left[\begin{array}{c}
x_{1} \\
\vdots \\
x_{6}
\end{array}\right]_{6 \times 1}
\end{aligned}
$$

\section{Model Verification}

Before starting the simulation study, the created mathematical model was compared with two previous numerical studies and the results are shown in Tables II and III. In [28], considering a cantilever beam divided into four finite elements, four different models were created by placing piezoelectric elements in different positions and the dominant first two vibration mode natural frequency values were obtained for each model. These values were compared with the values obtained from the current study in Table II. The mechanical and physical properties of the considered beam are given in [28]. In [29], a bimorph cantilever beam consisting of two piezoelectric PVDF layers divided into five finite elements, was considered and tip displacements were obtained by applying various voltage values in the range of $0-200 \mathrm{~V}$. The dimensions of the PVDF beam are $L=100 \mathrm{~mm}, w=5 \mathrm{~mm}$ and $h=1 \mathrm{~mm}$. The tip displacement values were compared with the values obtained from the current study in Table III. The mechanical properties of the PVDF layers are given in [29].

TABLE II. NATURAL FREQUENCY COMPARISON

\begin{tabular}{|l|l|l|c|c|}
\hline \multirow{2}{*}{ Model } & \multicolumn{4}{|c|}{ Natural frequency (Hz) } \\
\cline { 2 - 5 } & \multicolumn{3}{|c|}{$[\mathbf{2 8}]$} & \multicolumn{2}{c|}{ Present } \\
\cline { 2 - 5 } & Mode 1 & Mode 2 & Mode 1 & Mode 2 \\
\hline Model 1: Fixed end & 6.66 & 36.89 & 6.6581 & 36.8735 \\
\hline Model 2 & 4.73 & 27.43 & 4.7246 & 27.4143 \\
\hline Model 3 & 3.85 & 30.52 & 3.8212 & 30.2934 \\
\hline Model 4: Free end & 3.16 & 25.34 & 3.0433 & 25.0924 \\
\hline
\end{tabular}

TABLE III. TIP DISPLACEMENT COMPARISON

\begin{tabular}{|c|c|c|}
\hline \multirow{2}{*}{ Voltage (V) } & \multicolumn{2}{|c|}{ Tip displacem ents $\left(\times \mathbf{1 0}^{-4} \mathbf{m}\right)$} \\
\cline { 2 - 3 } & {$[\mathbf{2 9}]$} & Present \\
\hline 50 & 0.1755 & 0.1736 \\
\hline 100 & 0.3409 & 0.3377 \\
\hline 150 & 0.5067 & 0.4991 \\
\hline 200 & 0.6819 & 0.6706 \\
\hline
\end{tabular}

\section{LQR CONTROL DESIGN}

LQR control theory is used in active vibration control of smart cantilever beams. The cost function can be written as [30]:

$$
J=\frac{1}{2} \int_{0}^{\infty}\left(x^{T} Q x+u^{T} R u\right) d t
$$

where $[Q]$ and $[R]$ are the positive semi-definite symmetric and positive definite symmetric weighting matrices respectively. Larger $[Q]$ means that a better control performance is requested from the controller. On the other hand, the meaning of the larger $[R]$ is the reduction of the permissible control effort. Using the linear control law, the control input can be found in:

$$
u=-K x
$$

where $K$ is the feedback control gain which can be obtained as:

$$
K=R^{-1} B^{T} P
$$

where $P$ is a algebraic Ricatti equation solution of (54):

$$
P A+A^{T} P-P B R^{-1} B^{T} P=0
$$

Once $P$ is calculated, the actuator input voltage can be found with the help of (27). $[Q]$ and $[R]$ matrices can be written as:

$$
\begin{aligned}
& {\left[Q_{i i}\right]=\left[\begin{array}{cccc}
q_{11} & 0 & 0 & 0 \\
0 & \ddots & 0 & 0 \\
0 & 0 & \ddots & 0 \\
0 & 0 & 0 & q_{n n}
\end{array}\right]} \\
& {\left[R_{i i}\right]=\left[\begin{array}{cccc}
r_{11} & 0 & 0 & 0 \\
0 & \ddots & 0 & 0 \\
0 & 0 & \ddots & 0 \\
0 & 0 & 0 & r_{n n}
\end{array}\right]}
\end{aligned}
$$

The $[Q]$ and $[R]$ weighting matrices are obtained using Bryson's method [31]:

$$
q_{i i}=\left(\frac{1}{\left|x_{i}\right|_{\max }^{2}}\right), r_{i i}=\left(\frac{1}{\left|u_{i}\right|_{\max }^{2}}\right) \quad i=1,2,3, ., n
$$

In the Bryson's method, in a system with $n$ state variables, the state weight coefficient of each state variable is determined according to the maximum acceptable error amount of that variable $\left(\left|x_{i}\right|_{\max }\right)$. The determination of the $R$ control weight coefficient depends on the maximum acceptable value of the control signal $\left(\left|u_{i}\right|_{\max }\right)$.

\section{RESULTS AND DISCUSSION}

As shown in Figure 3, the beam was divided into ten finite elements and the actuator-sensor pair was placed in three different positions to examine the effect of the pair's position on the beam. As a result, three different models were created, and a state space model was obtained for each model. Finite element model and LQR controller were programmed in MATLAB. The maximum acceptable error was $2 \%$ and the maximum voltage applied to the actuator was $200 \mathrm{~V}$. The feedback control gain $K$ obtained for each model can be written as:

$$
\begin{aligned}
& {[K]_{1}=\left[\begin{array}{llllll}
-25.3 & 146.7 & -129.2 & 355.2 & 95.1 & 28.6
\end{array}\right]} \\
& {[K]_{2}=\left[\begin{array}{llllll}
52.2 & 434.8 & 326.6 & -426.3 & 145.9 & 37.7
\end{array}\right]}
\end{aligned}
$$




$$
[K]_{3}=\left[\begin{array}{llllll}
53.8 & 410.4 & 364.5 & -432.1 & 166.6 & 42.1
\end{array}\right]
$$

The natural frequencies for the first three vibration modes of the three models are given in Table IV. The step responses for the first three vibration modes of the system are given in Figures 4-6.

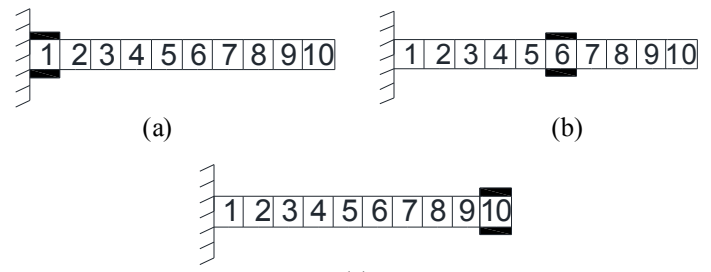

(c)

Fig. 3. Position of the PZT sensors. (a) model 1, (b) model 2, (c) model 3.

TABLE IV. NATURAL FREQUENCIES

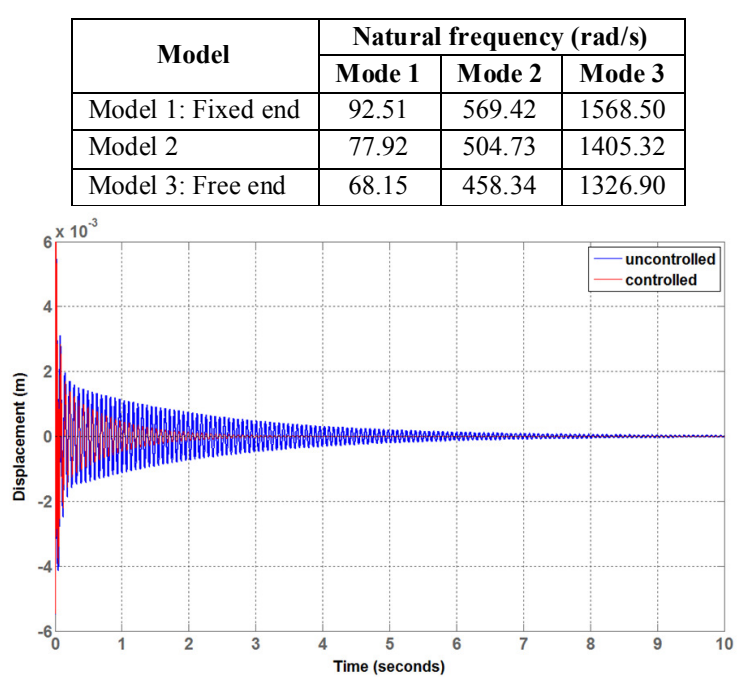

Fig. 4. Closed loop displacement responses of model 1.

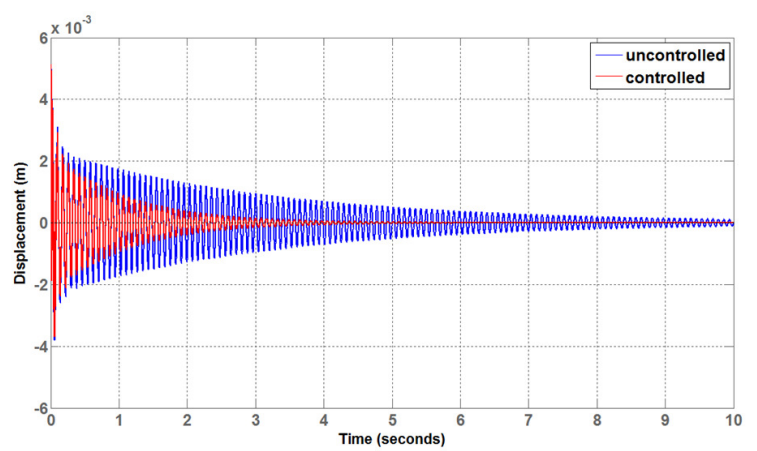

Fig. 5. Closed loop displacement responses of model 2.

When Table IV and Figures 4-6 are examined, it is seen that as the actuator-sensor pair moves from the fixed end to the free end, the stiffness of the system increases depending on the natural frequency and thus the vibration amplitudes and settling time decrease. The required actuator voltage for the vibration control of the three models is shown in Figures 7-9.

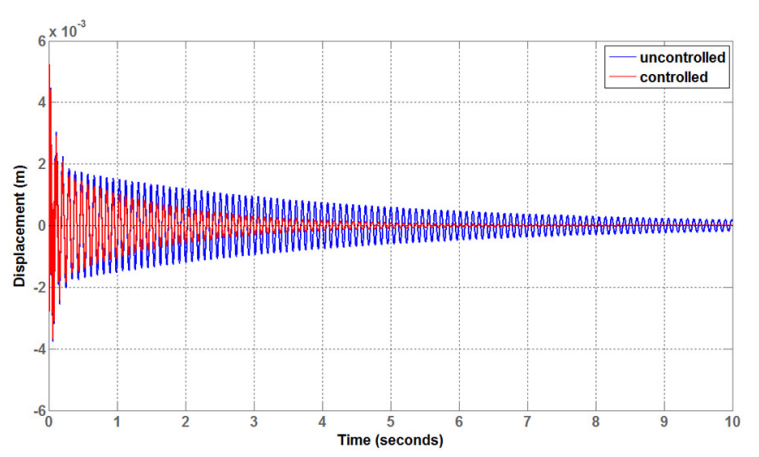

Fig. 6. Closed loop displacement responses of model 3.

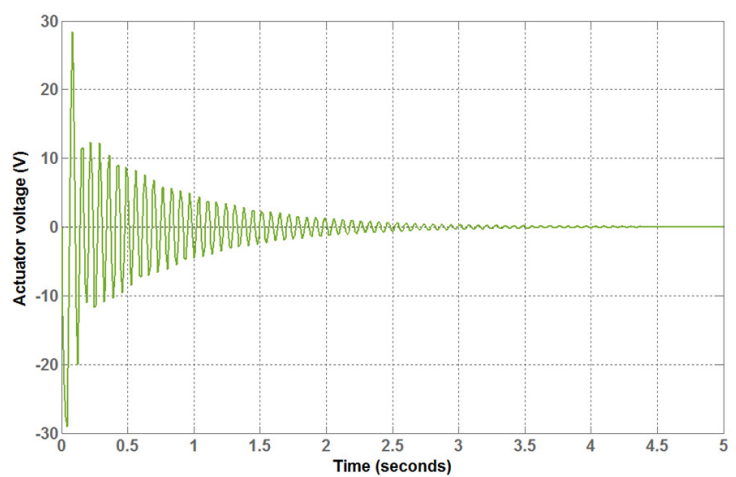

Fig. 7. Maximum actuator voltage requirement of model 1.

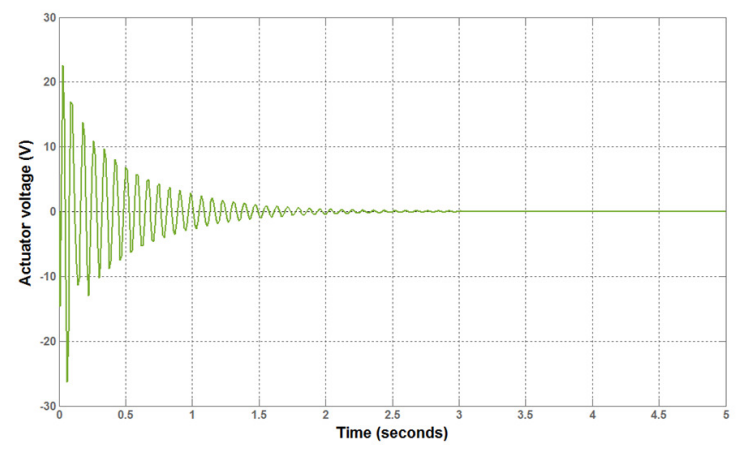

Fig. 8. Maximum actuator voltage requirement of model 2.

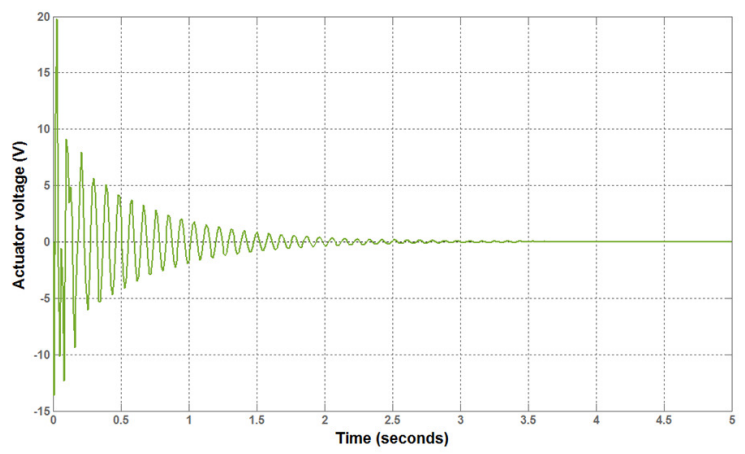

Fig. 9. Maximum actuator voltage requirement of model 3.

The obtained settling time and actuator voltage values, according to the simulation results are shown in Table V. 
TABLE V. SETTLING TIME AND ACTUATOR VOLTAGE

\begin{tabular}{|l|c|c|}
\hline \multicolumn{1}{|c|}{ Model } & Settling time (s) & Maximum Voltage (V) \\
\hline Model 1: Fixed end & 2.33 & 28.05 \\
\hline Model 2 & 3.39 & 23.12 \\
\hline Model 3: Free end & 4.56 & 19.88 \\
\hline
\end{tabular}

As seen in Table $\mathrm{V}$, the best settling time values were obtained in model 1. Although the required actuator voltage in model 1 is higher than that of other models, the difference is not significant when settling time values are considered.

\section{CONCLUSION}

This paper presents the active vibration control of a smart flexible cantilever beam bonded with a collocated PZT actuator and sensor pair. An LQR controller was designed for the control of first three vibration modes of the beam. The natural frequency and tip displacement values obtained to verify the finite element model and equations of motion created within the scope of the study were compared with existing studies in the literature. It has been determined that the natural frequency values are similar with a maximum error rate of $0.98 \%$ and the tip displacement values are similar to the $1.66 \%$ error rate. The position of the PZT sensor-actuator pair on the beam was changed to optimally determine the sensor-actuator position. As a result of the simulation study, system response, settling time, and required actuator voltage were determined for the first three vibration modes. The simulation results demonstrate that the best vibration damping results were obtained when the PZT actuator and sensor were affixed to the fixed end.

\section{REFERENCES}

[1] T. C. Manjunath and B. Bandyopadhyay, "Vibration control of a smart structure using periodic output feedback technique," Asian Journal of Control, vol. 6, no. 1, pp. 74-87, Mar. 2004, https://doi.org/10.1111/ j.1934-6093.2004.tb00185.x.

[2] A. M. Morad, A. Elzahaby, S. Abdallah, M. Kamel, and M. K. Khalil, "Application of Piezoelectric Materials for Aircraft Propeller Blades Vibration Damping," International Journal of Scientific \& Engineering Research, vol. 6, no. 8, pp. 513-520, Aug. 2015.

[3] M. Dadfarnia, N. Jalili, Z. Liu, and D. M. Dawson, "An observer-based piezoelectric control of flexible Cartesian robot arms: theory and experiment," Control Engineering Practice, vol. 12, no. 8, pp. 10411053, Aug. 2004, https://doi.org/10.1016/j.conengprac.2003.09.003.

[4] C. Chen, A. Sharafi, and J. Sun, "A high density piezoelectric energy harvesting device from highway traffic - Design analysis and laboratory validation," Applied Energy, vol. 269:115073, Jul. 2020, https://doi.org/ 10.1016/j.apenergy.2020.115073.

[5] K. Yang, J. Zhu, M. Wu, and W. Zhang, "Integrated optimization of actuators and structural topology of piezoelectric composite structures for static shape control," Computer Methods in Applied Mechanics and Engineering, vol. 334, pp. 440-469, Jun. 2018, https://doi.org/ 10.1016/j.cma.2018.01.021.

[6] M. M. Jovanović et al., "Experimental investigation of spillover effect in system of active vibration control," FME Transactions, vol. 42, no. 4, pp. 329-334, Nov. 2014, https://doi.org/10.5937/fmet1404329J.

[7] M. J. Balas, "Active control of flexible systems," Journal of Optimization Theory and Applications, vol. 25, no. 3, pp. 415-436, Jul. 1978, https://doi.org/10.1007/BF00932903.

[8] M. J. Balas, "Feedback control of flexible systems," IEEE Transactions on Automatic Control, vol. 23, no. 4, pp. 673-679, Aug. 1978, https://doi.org/10.1109/TAC.1978.1101798.

[9] S. M. Joshi, Control of Large Flexible Space Structures. Berlin: SpringerVerlag, 1989.
[10] J.L. Fanson and T. K. Caughey, "Positive Position Feedback Control for Large Space Structures," AIAA Journal, vol. 28, no. 4, pp. 717-724, Apr. 1990, https://doi.org/10.2514/3.10451.

[11] G. Song, P. Z. Qiao, W. K. Binienda, and G. P. Zou, "Active Vibration Damping of Composite Beam using Smart Sensors and Actuators," Journal of Aerospace Engineering, vol. 15, no. 3, pp. 97-103, Jul. 2002, https://doi.org/10.1061/(ASCE)0893-1321(2002)15:3(97).

[12] B. Sung, E. Lee, and I. Kim, "Displacement Control of Piezoelectric Actuator using the PID Controller and System Identification Method," in 2008 Joint International Conference on Power System Technology and IEEE Power India Conference, 2008, pp. 1-7, https://doi.org/10.1109/ ICPST.2008.4745381.

[13] B. Ding, Y. Li, X. Xiao, and Y. Tang, “Optimized PID Tracking Control for Piezoelectric Actuators based on the Bouc-Wen Model Bingxiao," in 2016 IEEE International Conference on Robotics and Biomimetics, 2016, pp. 1576-1581, https://doi.org/10.1109/ROBIO.2016.7866552.

[14] D. Sun, J. K. Mills, J. Shan, and S. K. Tso, “A PZT actuator control of a single-link flexible manipulator based on linear velocity feedback and actuator placement," Mechatronics, vol. 14, no. 4, pp. 381-401, May 2004, https://doi.org/10.1016/S0957-4158(03)00066-7.

[15] Z. Qiu, X. Zhang, H. Wu, and H. Zhang, "Optimal placement and active vibration control for piezoelectric smart flexible cantilever plate," Journal of Sound and Vibration, vol. 301, no. 3-5, pp. 521-543, Apr. 2007, https://doi.org/10.1016/j.jsv.2006.10.018.

[16] P. Shimon, E. Richer, and Y. Hurmuzlu, "Theoretical and experimental study of efficient control of vibrations in a clamped square plate," Journal of Sound and Vibration, vol. 282, no. 1-2, pp. 453-473, Apr. 2005, https://doi.org/10.1016/j.jsv.2004.02.057.

[17] J. Zhang, L. He, E. Wang, and R. Gao, "The Design of LQR Controller Based on Independent Mode Space for Active Vibration Control," Advances in Computation and Intelligence, vol. 5370, pp. 649-658, 2008, https://doi.org/10.1007/978-3-540-92137-0_71.

[18] M. Sareban, "Evaluation of Three Common Algorithms for Structure Active Control," Engineering Technology and Applied Science Research, vol. 7, no. 3, pp. 1638-1646, Jun. 2017, https://doi.org/ 10.48084/etasr. 1150 .

[19] T. Roy and D. Chakraborty, "GA-LQR Based Optimal Vibration Control of Smart FRP Composite Structures with Bonded PZT Patches," Journal of Reinforced Plastics and Composites, vol. 28, no. 11, pp. 1383-1404, Aug. 2009, https://doi.org/10.1177/0731684408089506.

[20] P. Shen, "Application of Genetic Algorithm Optimization LQR Weighting Matrices Control Inverted Pendulum," Applied Mechanics and Materials, vol. 543-547, pp. 1274-1277, Mar. 2014, https://doi.org/ 10.4028/www.scientific.net/AMM.543-547.1274.

[21] S. Ghoreishi, M. Nekoui, and S. Basiri, "Optimal Design of LQR Weighting Matrices based on Intelligent Optimization Methods," International Journal of Intelligent Information Processing, vol. 2, no. 1, Mar. 2011, https://doi.org/10.4156/IJIIP.VOL2.ISSUE1.7.

[22] W. J. Bottega, Engineering Vibrations, 2nd ed. Boca Raton, FL: CRC Press, 2006.

[23] F. P. Beer, E. R. Johnston, J. T. DeWolf, and D. F. Mazurek, Mechanics of Materials, 6th ed. New York, NY, USA: McGraw-Hill, 2012.

[24] J. Gryzagoridis, G. Oliver, and D. Findeis, "On the equivalent flexural rigidity of sandwich composite panels," Insight: Non-Destructive Testing and Condition Monitoring, vol. 57, no. 3. pp. 140-143, 2015, https://doi.org/10.1784/insi.2014.57.3.140.

[25] H. Almabrouk, G. Agnus, and Y. Bernard, "Geometry Investigation and Performance Optimization of a Single-Mass Piezoelectric 6-DOF IMU," Engineering Technology and Applied Science Research, vol. 10, no. 5, pp. 6282-6289, Oct. 2020, https://doi.org/10.48084/etasr.3711.

[26] “IEEE Standard on Piezoelectricity,” ANSI/IEEE Std .176-1987, 1988.

[27] A. K. Chopra, Dynamics of Structures: Theory and Applications to Earthquake Engineering, 5th ed. Englewood Cliffs, New Jersey, NJ, USA: Prentice Hall, 2017.

[28] B. Bandyopadhyay, T. Manjunath, and M. Umapathy, Modeling, Control and Implementation of Smart Structures: A FEM-State Space Approach. Berlin, Germany: Springer, 2007. 
[29] S. H. Chen, Z. D. Wang, and X. H. Liu, “Active vibration control and suppression for intelligent structures," Journal of Sound and Vibration, vol. 200, no. 2, pp. 167-177, 1997, https://doi.org/10.1006/ jsvi.1996.0694.

[30] D. J. Inman, Vibration with Control, 2nd Ed. Chichester, West Sussex, UK: John Wiley \& Sons Inc., 2017.

[31] A. E. Bryson and Y. C. Ho, Applied Optimal Control: Optimization, Estimation, and Control. Washington, USA: Hemisphere Publishing Corporation, 1975. 